\title{
A case of irritation fibroma
}

\author{
Meng Jiang, Wenbo Bu, Xu Chen, Heng Gu
}

Institute of Dermatology, Jiangsu Key Laboratory of Molecular Biology for Skin Diseases and STIs, Chinese Academy of Medical Science and Peking Union Medical College, Nanjing, China

Adv Dermatol Allergol 2019; XXXVI (1): 125-126

DOI: https://doi.org/10.5114/ada.2019.82834

A 53-year-old man was referred to the Dermatology Clinic with a 2-year history of an exophytic lesion on the tip of his tongue. He did not accept any treatment since the lesion had no subjective symptoms. The lesion developed slowly and showed no notable change in size until this visit. The patient was systemically healthy, but he had a long history of smoking for almost 30 years and at least 20 cigarettes per day. In addition, he denied any history of trauma in his oral cavity. The lesion showed a well-defined nodule with approximate $0.5 \mathrm{~cm}$ diameter on the tip of the tongue. The nodule was firm and presented a color resembling normal mucosa. The lesion was resected completely under local anaesthesia. The tissue sample of the lesion was subjected to histopathological examination. Histological features showed hyperkeratosis, hyperplasia of epidermis, proliferation of fibroblasts and hyaline degeneration in partial hyperplastic collagen. We observed vascular dilatation and proliferation within the hyperplastic fibrous connective tissue in the dermis and no infiltration of inflammatory cells (Figure 1). Conclusively, a diagnosis of irritation fibroma was made. No recurrence has occurred after 6 months' follow-up.

Irritation fibroma is an exophytic soft tissue mass in the oral mucosa. Indeed, it is not a real neoplasm but a focal hyperplasia of fibrous connective tissue induced by local trauma or chronic irritation [1, 2]. An earlier population-based epidemiological analysis showed that irritation fibroma was the second common benign oral lesion in Caucasians over 35 years of age [3]. The predilection sites were the buccal mucosa, labial and lingual surfaces. It also revealed that there was no prominent difference in the incidence rate between the sexes. However, data may vary from country to country regarding the occurrence of oral diseases in subsequent retrospective studies $[4,5]$.
Clinically, irritation fibromas usually manifest an exophytic, firm and asymptomatic nodule with pink or flesh-colored and smooth surface. The nodule often has a well-defined boundary. It is slow-growing and rarely exceeds $1.5 \mathrm{~cm}$ in diameter. The histopathological feature of irritation fibroma appears as a nodular mass composed of collagenized fibrous connective tissue. The epidermis usually presents hyperplasia and hyperkeratosis due to chronic irritation. Dense collagen fibers and focal hyperplasia of mature fibroblasts can be found in connective tissue, with slight or no inflammatory cell infiltration [6]. In view of the similarity of its clinical features with other oral lesions, reactive hyperplasia of fibrous connective tissue is difficult to be distinguished from the true neoplasms. Other lesions on the tongue including mucocele, neurilemmoma, neurofibroma and granular cell tumor can be ruled out due to respective histological features. In this case, the histopathological features of the lesion coincided with those of irritation fibroma. Complete excision is the recommended treatment for irritation fibroma. Other therapies including cryosurgery or intralesional injection of corticosteroids can be considered on condition that there is a definitive diagnosis based on histopathologic findings [7, 8].

This case exhibited the typical features of irritation fibroma, including the nodule with normal color, occurrence on the predilection site (tongue), chronic irritation (smoking) and proliferation of fibroblasts and hyperplastic collagen in histology. The patients with lesions in the buccal cavity, especially the tongue, are not rare in the dermatology clinics. However, some dermatologists might not pay attention to these diseases. Furthermore, many common skin diseases have the particular manifestation in the mucosa membrane in the oral cavity, such as lichen planus. Therefore, dermatologists should be encouraged to improve the ability to identify the oral diseases.

Address for correspondence: Xu Chen, Wenbo Bu, Heng Gu, Institute of Dermatology, Jiangsu Key Laboratory of Molecular Biology for Skin Diseases and STIS, Chinese Academy of Medical Science and Peking Union Medical College, 12 Jiangwangmiao St, Nanjing 210042, China, phone: +86 258547 8919, fax: +86 25 85414477, e-mail: doctor_chx@hotmail.com; buwenbo@163.com; doctor_guheng@hotmail.com. Received: 17.11 .2017 , accepted: 24.01.2018. 

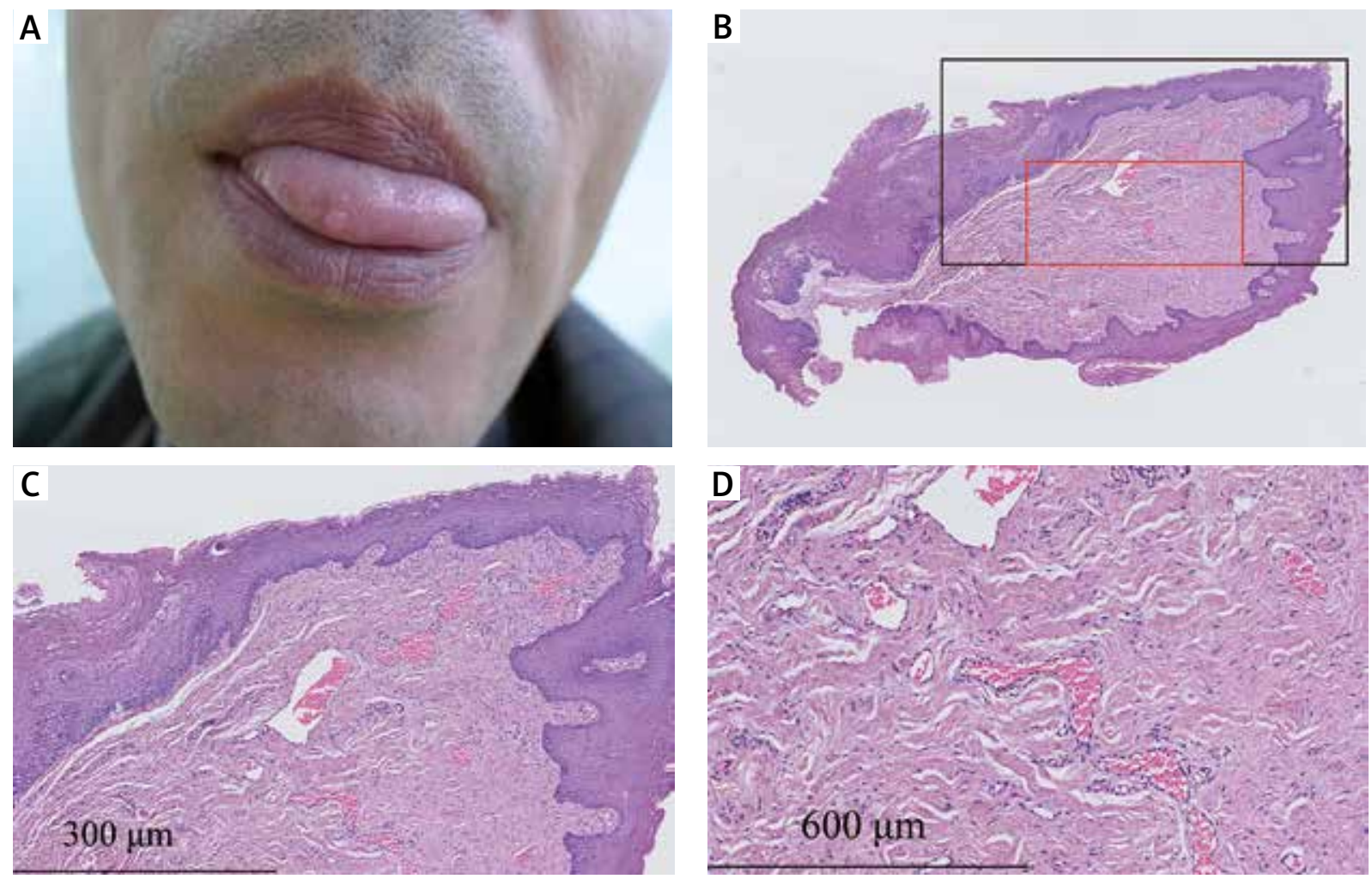

Figure 1. Clinical and histopathological photos of the lesion from the patient. A - Primary lesion on the tip of the patient's tongue. B - Histopathological appearance of the lesion had a polypoid shape. C - Epidermis was hyperplastic and hyperkeratotic. Increased and dilated vascular tissue? can be seen among the hyperplastic fibrous connective tissue. D - Dense collagen fibers and fibroblasts proliferation were presented in the dermis, with no inflammatory cell infiltration

\section{Acknowledgments}

This work was supported by CAMS Innovation Fund for Medical Sciences (2017-12M-1-017 and 2016-12M1-005). Xu Chen and Wenbo Bu are supported by the PUMC Youth Fund and Fundamental Research Funds for the Central Universities (3332015116, 3332014008, 2016RC320005 and 2016ZX320014).

\section{Conflict of interest}

The authors declare no conflict of interest.

\section{References}

1. Esmeili T, Lozada-Nur F, Epstein J. Common benign oral soft tissue masses. Dent Clin North Am 2005; 49: 223-40.

2. Naderi NJ, Eshghyar N, Esfehanian H. Reactive lesions of the oral cavity: a retrospective study on 2068 cases. Dent Res 2012; 9: 251-5.

3. Bouquot JE, Gundlach KK. Oral exophytic lesions in 23,616 white Americans over 35 years of age. Oral Surg Oral Med Oral Pathol 1986; 62: 284-91.

4. Dhanuthai K, Rojanawatsirivej S, Somkotra T, et al. Geriatric oral lesions: a multicentric study. Geriatr Gerontol Int 2016; 16: 237-43.
5. Alaeddini M, Barghammadi R, Eshghyar N, et al. An analysis of biopsy-proven tongue lesions among 8,105 dental outpatients. J Contemp Dent Pract 2014; 15: 1-7.

6. Neville BW, Damm DD, Allen CM, et al. Oral and Maxillofacial Pathology. Saunders/Elsevier, Philadelphia, USA 2009.

7. Valério RA, de Queiroz AM, Romualdo PC, et al. Mucocele and fibroma: treatment and clinical features for differential diagnosis. Braz Dent J 2013; 24: 537-41.

8. Parwani S, Parwani RN. Diagnosis and management of focal reactive overgrowths of gingival: a case series. J Mich Dent Assoc 2014; 96: 36-47. 